\section{Assembler language software for linking the Apple II to a PDP-11 as a dumb VDU}

\author{
D. G. WASTELL \\ Department of Medical Computing, University of Manchester \\ Manchester M15 6FH, England
}

Linking microcomputers to larger machines for file transfer is commonplace. Having such links in place, it is useful also to have the micro serve as a dumb terminal. This note is concerned with linking Apple II micros to a host PDP-11 (running under RSX-11M), a hardware combination found in many psychological laboratories. Many asynchronous serial interfaces are available for Apples. Some have firmware allowing a dumb-terminal mode (e.g., the Apple communications card), and others do not. However, even when the firmware exists, it may not always provide an adequate facility. For instance, the RSX-11M operating system on the PDP-11 uses ASCII character 127 (DEL) as the rubout character. The Apple, on the other hand, uses the backspace character for this purpose (BS, ASCII 8), and DEL is not available at all from the keyboard. Another character, unavailable but essential for RSX-11M, is the lefthand square bracket (ASCII 91), although shift-M provides the right-hand one. A final problem is that the "new line" sequence issued under RSX-11M includes carriage returns as well as a line feed. The screen output subroutine in the Apple generates a linefeed at each carriage return. Unnecessary additional line-feeds are thus generated with each new line.

Because the firmware in the Apple communications card has no facility for character conversion, software was written to provide the extra features required for a satisfactory full-duplex dumb-terminal facility. This exercise was also instructive in using the Apple assembler and in gaining familiarity with the Apple monitor. Because our requirements were quite specific in both software (i.e., full-duplex terminal mode only) and hardware (slot number and data format fixed), the final program could also be kept simpler and more readable than the usual listings of interface software, which are rather daunting to the less sophisticated computer user. The listing is shown in Figure 1.

From the programmer's point of view, the serial interface constitutes two registers: one holding the status of the interface, and the other in which data are either received or put to be transmitted. Briefly, follow-

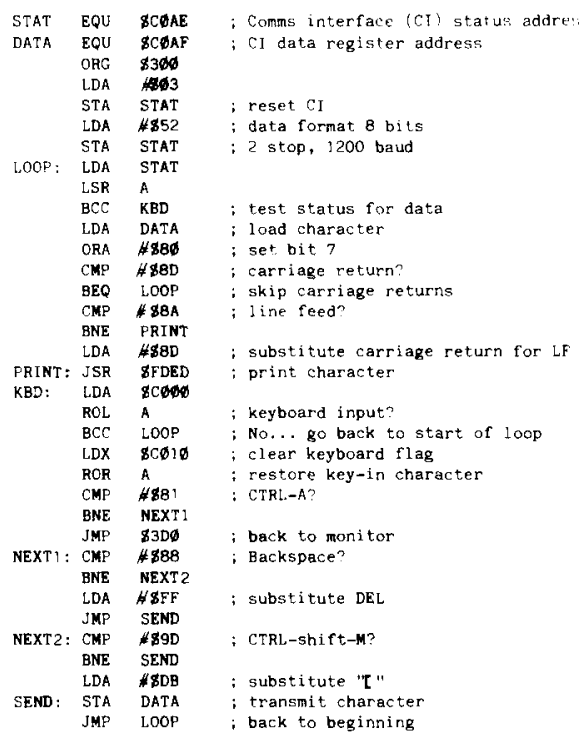

Figure 1. Assembler language program for dumb-terminal emulation.

ing resetting and initialization of the interface (here 8 bits, two stop, 1,200 baud), the program sits in a loop, first testing the interface status register for the arrival of data. Newly arrived characters are inspected before sending to the screen print routine. Any carriage returns are skipped, and a carriage return is substituted for any line feeds. A test is then made for any keyboard input. A control-B returns to the monitor. DEL is substituted if a backspace has been typed, and "[" is generated in response to CTRL-shift-M. The keyboard character is then output over the interface, and a return is made to the beginning of the loop. Because screen scrolling on the Apple is a software function, it takes time, and thus characters immediately received after a new line may be lost unless the RSX-11M terminal driver option for sending out padding nulls is selected.

This software performed well at 1,200 baud, and its writing was an instructive exercise. With small modifications, such a simple program may easily be adapted to different hardware arrangements (slot numbers, data formats, etc.) and to any serial interfaces embodying the two-register structure, particularly to those without any existing dumb terminal facility.

(Accepted for publication March 16, 1982.) 